\title{
Astrophysical imaging of Kerr black holes with scalar hair
}

\author{
F. H. Vincent, ${ }^{1, *}$ E. Gourgoulhon, ${ }^{2, \dagger}$ C. Herdeiro, ${ }^{3, *}$ and E. Radu ${ }^{3, \$}$ \\ ${ }^{1}$ LESIA, CNRS UMR 8109, Observatoire de Paris, Université Pierre et Marie Curie, \\ Université Paris Diderot, 5 place Jules Janssen, 92190 Meudon, France \\ ${ }^{2}$ LUTH, CNRS UMR 8102, Observatoire de Paris, Université Paris Diderot, 5 place Jules Janssen, \\ 92190 Meudon, France \\ ${ }^{3}$ Departamento de Física da Universidade de Aveiro and Centre for Research and Development \\ in Mathematics and Applications (CIDMA), Campus de Santiago, 3810-183 Aveiro, Portugal
}

(Received 17 June 2016; published 26 October 2016)

\begin{abstract}
We address the astrophysical imaging of a family of deformed Kerr black holes (BHs). These are stationary, asymptotically flat $\mathrm{BH}$ spacetimes that are solutions of general relativity minimally coupled to a massive, complex scalar field: Kerr BHs with scalar hair (KBHsSH). Such BHs bifurcate from the vacuum Kerr solution and can be regarded as a horizon within a rotating boson star. In a recent letter [P. V. P. Cunha, C. A. R. Herdeiro, E. Radu, and H. F. Rúnarsson, Phys. Rev. Lett. 115, 211102 (2015).], it was shown that $\mathrm{KBHsSH}$ can exhibit very distinct shadows from the ones of their vacuum counterparts. The setup therein, however, considers the light source to be a celestial sphere sufficiently far away from the BH. Here, we analyze KBHsSH surrounded by an emitting torus of matter simulating a more realistic astrophysical environment, and study the corresponding lensing of light as seen by a very faraway observer, to appropriately model ground-based observations of Sgr $\mathrm{A}^{*}$. We find that the differences in imaging between $\mathrm{KBHsSH}$ and comparable vacuum Kerr BHs remain, albeit less dramatic than those observed for the corresponding shadows in the previous setup. In particular, we highlight two observables that might allow differentiating KBHsSH and Kerr BHs. The first is the angular size of the photon ring (in a Kerr spacetime) or lensing ring (in a KBHSH spacetime), the latter being significantly smaller for sufficiently non-Kerr-like spacetimes. The second is the existence of an edge in the intensity distribution (the photon ring in Kerr spacetime). This edge can disappear for very non-Kerr-like KBHsSH. It is plausible, therefore, that sufficiently precise very long baseline interferometric observations of $\mathrm{BH}$ candidates can constrain this model.
\end{abstract}

DOI: $10.1103 /$ PhysRevD.94.084045

\section{INTRODUCTION}

Over the last half a century, electromagnetic observations have gathered a solid body of evidence for the existence of astrophysical black holes (BHs) —see e.g. [1]. Recently, the detection of the gravitational wave event GW150914 [2] has strengthened the case for astrophysical BHs, now using a completely independent channel. Observations with ever-increasing precision, both in the gravitational wave and electromagnetic channels, are expected over the next decade, leading to a more thorough understanding of the true nature of astrophysical $\mathrm{BH}$ candidates and, in particular, discriminating the paradigmatic BHs of general relativity (GR) from more exotic alternatives [3]. It is thus quite timely to obtain the appropriate phenomenology for any theoretically sound alternative model.

Steady-state BHs in vacuum GR have been recognized to be surprisingly simple since the 1970s: they have only

\footnotetext{
frederic.vincent@obspm.fr †eric.gourgoulhon@obspm.fr herdeiro@ua.pt

§eugen.radu@ua.pt
}

2 degrees of freedom [4-6] and are described by the elegant Kerr metric [7]. ${ }^{1}$ This geometry provides the standard model to obtain astrophysical phenomenology for $\mathrm{BH}$ candidates. Yet, assuming a priori the Kerr metric introduces an undesirable theoretical bias. Indeed, the Kerr solution is unique in vacuum, but different $\mathrm{BH}$ solutions may exist when considering generic types of matter (or departing from GR). Three main avenues are being investigated to avoid this bias. The first is to study geometries where one introduces, ad hoc, deformations of the Kerr geometry (see e.g. [8-10]), and investigate how much observations can constrain such deformations. A second avenue corresponds to attempts of studying generic black-hole spacetimes under some sufficiently broad parametrization scheme, with Schwarzschild and Kerr being particular realizations of the general framework, rather than fiducial solutions [11,12]. These two first approaches may be informative, but have the unsatisfactory feature that the deformed geometries, in general, do not have a clear origin as solutions of any sensible

\footnotetext{
${ }^{1}$ Here we are taking the viewpoint that astrophysical BHs are essentially electrically neutral.
} 
model. A third and more satisfactory first-principles approach, on the other hand, i.e. starting from a concrete GR plus a (physically reasonable and astrophysically plausible) matter model is hampered by the obvious difficulty in finding qualitatively new $\mathrm{BH}$ solutions, let alone solutions which are deformations of the vacuum Kerr BH.

Unexpectedly, in the last two years, new families of deformed Kerr BHs could be obtained within simple matter models. The first (and simplest) such family that was discovered, corresponds to Kerr BHs with scalar hair (KBHsSH) [13] (see also [14,15]). These are solutions of Einstein's gravity minimally coupled to a massive, complex, scalar field. This matter content obeys all energy conditions, and the solutions are regular on and outside an event horizon, thus providing a theoretically consistent model. Besides the vacuum limit, wherein they reduce to a subset of vacuum Kerr BHs, KBHsSH have also a solitonic limit, in which they reduce to well-known gravitating solitons known as boson stars [16,17]. KBHsSH have been found numerically, but a formal proof of their existence was given in [18]. Generalizations with selfinteractions were constructed in $[19,20]$ and with massive Proca fields in [21].

$\mathrm{KBHsSH}$ inherit a well-known property from boson stars: the maximal Arnowitt-Deser-Misner (ADM) mass possible is of the order of the Compton wavelength of the scalar field. This demands, for the existence of either boson stars or KBHsSH with ADM masses of the order of the solar mass, or higher, that the scalar field must be ultralight, with a mass of order of $10^{-10} \mathrm{eV}$, or smallersee the discussion in [20] for models including selfinteractions. Such ultralight particles are not present in the standard model of particle physics, but have been predicted in beyond standard model scenarios [22]. Thus, evidence for boson stars or KBHsSH could be faced as evidence for an ultralight particle beyond the standard model. Claiming such evidence, however, will only be possible if the phenomenology of these exotic compact objects is understood in detail and smoking guns that distinguish them from standard compact objects are identified.

Within the electromagnetic channel, a promising class of observations pertains to the lensing of light in the neighborhood of some supermassive $\mathrm{BH}$ candidates, together with their shadows [23]. The shadow of a BH is the region in the observer's sky comprising the directions of photons that asymptotically approach the event horizon in a backward ray-tracing computation. This type of observable is being targeted by the Event Horizon Telescope (EHT) [24], a millimeter-wavelength very long baseline interferometry network, which has the potential to test the Kerr paradigm and constrain alternative models [25]. In particular, the EHT will be able to image the shadow of the supermassive compact object at the center of the Galaxy, Sgr A*. The resolution of the instrument will reach $\approx 20 \mu$ as, which is smaller than the typical size of Sgr A*'s shadow, $\approx 50 \mu$ as.

In a recent letter [26], it was shown that the shadows of $\mathrm{KBHsSH}$ can be sharply distinct from the ones of the vacuum Kerr BHs, in some regions of the parameter space. This study, however, used a setup (first introduced in [27]) which had a primary goal of producing visually striking images of the lensing, and the corresponding differences with respect to Kerr, rather than simulating a realistic astrophysical environment. Thus, a natural question is whether introducing a more realistic astrophysical environment masks the peculiar shadows obtained in [26]. The main purpose of the current paper is to address this question.

We shall consider the same configurations of $\mathrm{KBHsSH}$ studied in detail in [26] and study their lensing, but with three main differences with respect to the former study. First, the ray tracing will be done with a well-tested code (GYото [28]), completely independent from that used in [26]. Second, the BHs are surrounded by an accretion torus, using the same model studied recently around Kerr BHs [29] and boson stars [30]. This is the light source, rather than a faraway celestial sphere, as in the setup considered in [26]. Third, we consider the observer to be at a realistic distance to model a ground-based observation of Sgr A*, whereas the observations collected in [26] were from the viewpoint of a much closer observer. Our main conclusion is that, even with this more realistic astrophysical setup, observable differences remain between the hairy BHs and their vacuum counterparts, even though the astrophysical environment partly masks the strikingly different shapes observed in [26]. More concretely, the total flux difference in the imaging of $\mathrm{KBHsSH}$ and comparable Kerr BHs is small for the sample of cases analyzed, but the photon ring size difference can have an observable signature. In more extreme cases the shadow, in the astrophysical setup, is essentially erased, in sharp contrast to that of the comparable Kerr BH.

This paper is organized as follows. In Sec. II we briefly review $\mathrm{KBHsSH}$ and, in particular, the sample of backgrounds to be addressed in this paper. In Sec. III we benchmark the use of GYOTо by comparing its results with the ones previously obtained for the shadows of KBHsSH. In Sec. IV we introduce the astrophysical environment around $\mathrm{KBHsSH}$ and produce the corresponding imaging. We conclude in Sec. V with a summary of our results and an outlook.

\section{THE BLACK-HOLE BACKGROUNDS}

The simplest KBHsSH are solutions of the EinsteinKlein-Gordon model, where the scalar field is free (no self-interactions), complex and massive (see [19,20] for generalizations including self-interactions). This model is described by the action: 
TABLE I. KBHsSH configurations considered in the present study. $M$ is the ADM mass, $M_{\mathrm{H}}$ is the horizon's Komar mass, $J$ is the total Komar angular momentum and $J_{\mathrm{H}}$ is the horizon's Komar angular momentum.

\begin{tabular}{lcccccccc}
\hline \hline & $M$ & $M_{\mathrm{H}}$ & $J$ & $J_{\mathrm{H}}$ & $\frac{M_{\mathrm{H}}}{M}$ & $\frac{J_{\mathrm{H}}}{J}$ & $\frac{J}{M^{2}}$ & $\frac{J_{\mathrm{H}}}{M_{\mathrm{H}}^{2}}$ \\
\hline Configuration I & $0.415 \mathcal{M}$ & $0.393 \mathcal{M}$ & $0.172 \mathcal{M}^{2}$ & $0.150 \mathcal{M}^{2}$ & $95 \%$ & $87 \%$ & 0.999 & 0.971 \\
Configuration II & $0.933 \mathcal{M}$ & $0.234 \mathcal{M}$ & $0.740 \mathcal{M}^{2}$ & $0.115 \mathcal{M}^{2}$ & $25 \%$ & $15 \%$ & 0.850 & 2.10 \\
Configuration III & $0.975 \mathcal{M}$ & $0.018 \mathcal{M}$ & $0.85 \mathcal{M}^{2}$ & $0.002 \mathcal{M}^{2}$ & $1.8 \%$ & $2.4 \%$ & 0.894 & 6.20 \\
\hline \hline
\end{tabular}

$S=\int d^{4} x \sqrt{-g}\left[\frac{R}{16 \pi G}-\frac{g^{\alpha \beta}}{2}\left(\Phi_{, \alpha}^{*} \Phi_{, \beta}+\Phi_{, \beta}^{*} \Phi_{, \alpha}\right)-m^{2} \Phi^{*} \Phi\right]$

Throughout we use units with $c=1=\hbar$. This model has two constants, Newton's constant $G$ and the scalar field mass $m$. We take the two associated natural scales to be Planck's mass $m_{P}=G^{-1 / 2}$, and the mass scale for boson stars/KBHsSH, which reads

$$
\mathcal{M}=\frac{m_{P}^{2}}{m}
$$

Indeed, the maximal ADM mass for boson stars/KBHsSH is $\alpha \mathcal{M}$, where $\alpha$ is a constant of order unity that depends on the particular type of boson stars-see [20,31] for a sample of concrete $\alpha$ values.

The hairy Kerr BH solutions are found with the following metric Ansatz for a stationary axisymmetric and circular spacetime:

$$
\begin{aligned}
d s^{2}= & -e^{2 F_{0}} N d t^{2}+e^{2 F_{1}}\left(\frac{d r^{2}}{N}+r^{2} d \theta^{2}\right) \\
& +e^{2 F_{2}} r^{2} \sin ^{2} \theta(d \varphi-W d t)^{2}
\end{aligned}
$$

where

$$
N \equiv 1-\frac{r_{H}}{r},
$$

$r_{H}$ being a constant (representing the radial coordinate of the event horizon), and $F_{1}, F_{2}$ and $W$ are functions of the spheroidal coordinates $(r, \theta)$. The vacuum Kerr metric can be written in this coordinate system. The explicit form of the coefficients can be found in [15,21]. Note that the parameters $b$ (in [21]) and $c_{t}$ (in [15]) relate as $b=-c_{t}$. In the following we shall dub these as spheroidal prolate $(S P)$ coordinates, cf. Appendix A of [21].

$\mathrm{KBHsSH}$ form a countable number of families labeled by the azimuthal harmonic index and the number of nodes in the scalar field. Here we shall focus on a particular member of this family, with the lowest azimuthal harmonic index (equal to 1) and the lowest number of nodes (no nodes - see [15] for a discussion of the general case). The latter defines fundamental states; excited solutions are likely unstable, towards decay into fundamental solutions, as in the case of boson stars [32]. The corresponding scalar field Ansatz is

$$
\Phi(t, r, \theta, \phi)=e^{-i w t} e^{i \varphi} \phi(r, \theta),
$$

where $w$ is a constant. In the following we shall address three particular solutions of the model (1), with the Ansatzë (3)-(5), which are dubbed configurations I-III in [26]. The numerical data for these solutions are publicly available [33]. Table I provides a brief description of the physical parameters of these configurations (from [26]).

These three KBHsSH configurations are compared to Kerr BH solutions with the same values of ADM mass and total angular momentum. These comparable Kerr solutions are denoted $\mathrm{Kerr}_{=\mathrm{ADM}}$ in [26]. They are comparable observationwise, because observations typically give access to the parameters $M$ and $J$.

Configuration I is a "rather Kerr-like" KBHSH. Only 5\% of the mass and $13 \%$ of angular momentum are stored in the scalar field. The horizon is also Kerr-like, in the sense that the Kerr bound is obeyed in terms of horizon quantities - a property which is not mandatory for other KBHsSH [34]. Its Kerr-like shadow's average radius is only a few percent smaller than that of its comparable Kerr counterpart. The latter is an almost extremal $\mathrm{BH}$, with $j \equiv J / M^{2}=0.999$.

Configuration II is a "not-so-Kerr-like" KBHSH. In this case, the majority of the mass $(75 \%)$ and angular momentum $(85 \%)$ are stored in the scalar field. The horizon is nonKerr-like, in the sense that it violates the Kerr bound in terms of horizon quantities. Its shadow is not only $25 \%$ smaller than that of the comparable Kerr BH [26], but it has also a peculiar shape-more square-than that observed for any vacuum Kerr BH. The comparable Kerr BH is less extremal than the corresponding one for configuration I, with $j=0.85$.

Finally, configuration III is a "very non-Kerr-like" KBHSH. Almost all mass (98.2\%) and angular momentum $(97.6 \%)$ are stored in the scalar field. The horizon is very non-Kerr-like, violating the Kerr bound in terms of horizon quantities by a factor of 6 . The horizon shape is quite exotic -it is delimited by a nonconvex curve and there are multiple disconnected shadows. The comparable Kerr BH has $j=0.894$.

In the following two sections we will readdress these configurations, first recomputing their shadows using 
GYOTO, and comparing the results with those previously obtained, and then performing their imaging in the astrophysical setup.

\section{RAY-TRACING SETUP AND SHADOW COMPUTATIONS}

We use in this article the ray-tracing code GYOTо, which is open-source software $[28,35]$. We employ this code to integrate numerically null geodesics in different $\mathrm{KBHsSH}$ numerical spacetimes and integrate the radiative transfer equation inside an accretion structure surrounding the $\mathrm{BH}$. The geodesic integration is performed backward in time from a distant observer. In our setup, the observer is located at a radial coordinate corresponding to the distance between Earth and the Galactic center, i.e. $8.33 \mathrm{kpc}$ [36]. The $\mathrm{KBHsSH}$ and Kerr solutions that we use are expressed in SP coordinates $(t, r, \theta, \varphi)$, as defined in [15]. In order to relate the SP radial coordinate $r$ expressed in units of $\mathcal{M}$, to a physical distance of $8.33 \mathrm{kpc}$, we need to fix the mass of Sgr A* Throughout this article we use $M=4.31 \times 10^{6} M_{\odot}$ [36], where $M$ is the ADM mass of the $\mathrm{BH}$. The integration is performed using a Runge-Kutta-Fehlberg adaptive-step integrator at order $7 / 8$ as implemented in the boost $\mathrm{C}++$ library. A recent study [37] has demonstrated the ability of GYOTO to ray trace accurately even over such very large distances. The observer is located at some fixed inclination $\theta=85^{\circ}$, where $\theta$ is the angle between the axis of rotation and the observer. This particular value of inclination is inherited from a previous study [29] where it was shown to be able to reproduce well the observed features of Sgr A* modeled as a Kerr BH. The observer is modeled by a screen, with every pixel corresponding to some direction of photon incidence. The total computed field of view is typically of $300 \mu$ as, unless otherwise stated. The various pixels of the screen are assigned with the value of the specific intensity transported by the photon corresponding to the pixel's direction of incidence. The output of the raytracing calculation is thus a map of specific intensity over some small field of view, which, in the following, will be called an image.

We use the numerical metrics corresponding to the $\mathrm{KBHsSH}$ and comparable Kerr solutions described in the previous section, which are publicly available [33]. We extended the LORENE library [38] to make it able to read these metrics and translate them to multidomain spectral grids. GYото is then able to perform ray tracing using such numerical spacetimes. The LORENE class $\mathrm{ScalarBH}$ used for generating GYOTO-compatible metrics from the $\mathrm{KBHsSH}$ raw data is publicly available in the latest version of LORENE. We remark that in preparing the framework for this study, we have compared rotating boson star solutions numerically generated by the KADATH code [39] in [31] with those generated by the code used in [13], FIDISOL/CADSOL [40], finding a very good agreement.

In this section, we want to compute the shadows of the three $\mathrm{KBHsSH}$, configurations I-III, and their comparable Kerr BHs. Our aim is first to determine what the shadows look like in an "astrophysically neutral" setup (i.e. no emission of electromagnetic radiation), which will be useful for discussing the astrophysically realistic images later on. We also want to compare our computations with the previous calculations developed in [26], to ensure consistency between the two completely independent ray-tracing codes. As a consequence, we do not consider any source of radiation and simply trace null geodesics until they approach the event horizon. The ray-traced images show only two intensity values: either 1 when the backtraced photon came arbitrarily close to the event horizon, or 0 otherwise.

Figure 1 shows the shadows of the KBHSH and comparable Kerr BH of our configuration I. They look very similar to Fig. 5, top-left and top-middle panels of [26]. Note, however, that therein an inclination of $\theta=90^{\circ}$
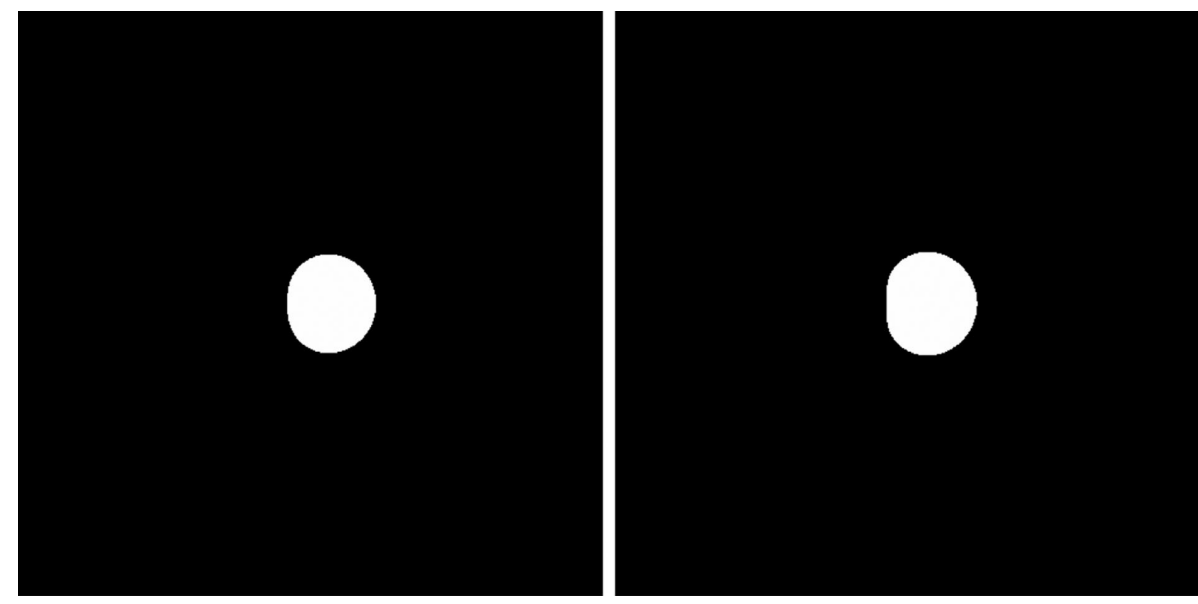

FIG. 1. Left: Shadow of the KBHSH of configuration I (field of view $300 \mu$ as). Right: The same for the comparable Kerr BH $\left(j_{\mathrm{ADM}}=0.999\right)$. 

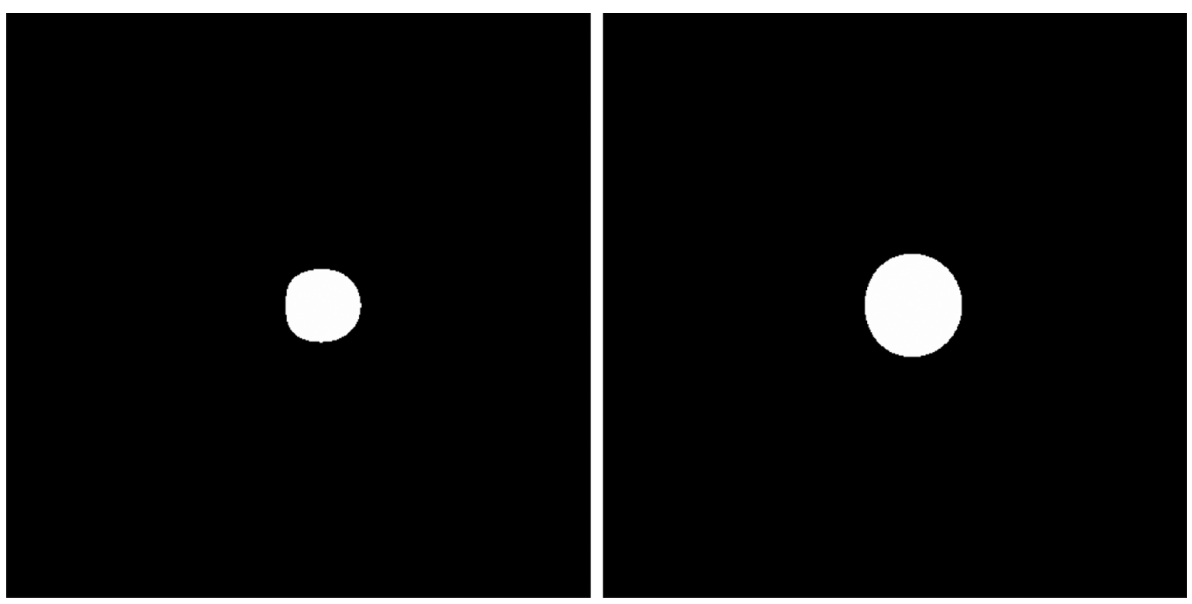

FIG. 2. Left: Shadow of the KBHSH of configuration II (field of view $300 \mu$ as). Right: The same for the comparable Kerr BH $\left(j_{\mathrm{ADM}}=0.85\right)$.

was used, whereas here we take $\theta=85^{\circ}$. The inclination impact is very mild, for this configuration, and the change in $\theta$ is barely noticeable in the shadow. Observe that in all solutions presented in this paper the BHs are rotating such that the left-hand side of the image is moving towards the reader.

Figure 2 shows the shadows of the KBHSH and comparable Kerr $\mathrm{BH}$ of our configuration II. They are very similar to the corresponding one in Fig. 5 of [26] (second line from the top). It is clear that the angular size of the shadow is smaller (by $\approx 25 \%$ ) for the KBHSH as compared to the comparable Kerr setup.

Figure 3 shows the shadows of the KBHSH and comparable Kerr BH of configuration III. The two shadows are extremely different, as already shown in [26]. The KBHSH shadow is rather different from the lower-left panel of Fig. 5 of [26], but the dominating difference is due to the change in inclination $\theta$ since our shadow is not symmetric with respect to the horizontal axis. In order to be able to compare more precisely with [26], Fig. 4 shows the shadow of the KBHSH of configuration III as observed from the equatorial plane $\left(\theta=90^{\circ}\right)$ and for a smaller field of view $(150 \mu \mathrm{as})$. The left panel shows the shadow as observed from Earth, while the right panel shows the shadow as observed by a very close observer, at the same radial coordinate as used in [26]. These two computations show that the topology of the shadow changes with the radial coordinate of the observer. The right panel of Fig. 4 is extremely similar to the lower-left panel of Fig. 5 of [26] thus validating the ray-tracing comparison in the most nonKerr-like spacetime.

\section{ACCRETION TORUS AND IMAGES}

We consider a simple toroidal accretion structure surrounding the various $\mathrm{BHs}$ of configurations I-III. This model is the same as presented in [29] and recently applied to boson stars [30]. It is based on the magnetized torus model of [41]. We will only present this accretion model very briefly and refer to [29] for details. The accretion torus
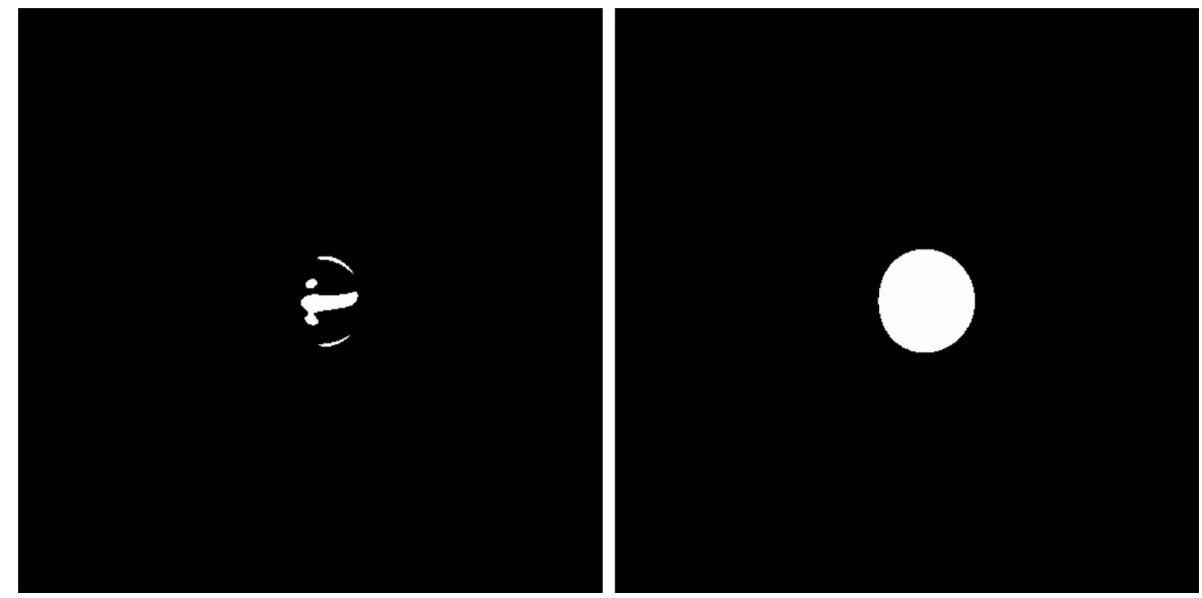

FIG. 3. Left: Shadow of the KBHSH of configuration III (field of view $300 \mu$ as). Right: The same for the comparable Kerr BH $\left(j_{\mathrm{ADM}}=0.894\right)$. 

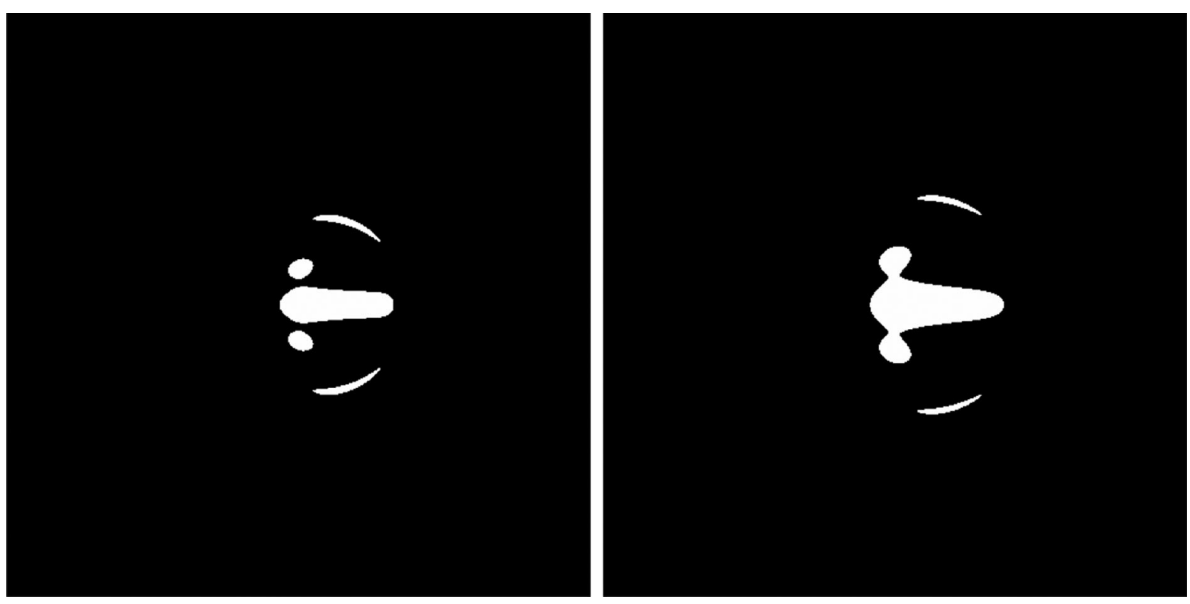

FIG. 4. Left: Shadow of the KBHSH of configuration III from an inclination of $\theta=90^{\circ}$ (field of view $150 \mu$ as). Right: Same computation, changing only the coordinate radius of the observer which is taken the same as in [26] (field of view $\pi / 2$ ). Observe that the shadow's topology depends on the observer's distance.

model that we consider is made of a non-self-gravitating perfect polytropic fluid circularly orbiting with constant specific angular momentum $\ell=-u_{\varphi} / u_{t}$, where $u$ is the fluid 4-velocity, which is completely fixed by imposing the constancy of $\ell$ and the circular motion. The original work of [41] considers a toroidal magnetic field. However, [30] showed that the synchrotron images are not sensitive to the direction of the magnetic field. We thus simplify the original model of [41] by considering an isotropized magnetic field. The energy-momentum conservation is readily integrated by considering a polytropiclike equation of state with the gas pressure $p$ and enthalpy $h$ (equal to the sum of the gas pressure and total energy density) related through

$$
p=\kappa h^{k},
$$

where $k$ is the polytropic exponent, and $\kappa$ is the polytropic constant. Conservation of energy-momentum then leads to

$$
W_{s}-W+\frac{k}{k-1} \kappa h^{k-1}=0,
$$

where $W=-\ln \left|u_{t}\right|$ is a potential (known throughout spacetime because the 4 -velocity is fixed once $\ell$ is chosen) and $W_{s}$ is its value at the surface of the torus. This immediately gives

$$
\begin{aligned}
& h=h_{c} \omega^{1 /(k-1)}, \\
& \kappa=\left(W_{c}-W_{s}\right) \frac{k-1}{k} h_{c}^{1-k},
\end{aligned}
$$

where $h_{c}$ is the central enthalpy, $\omega=\left(W-W_{s}\right) /\left(W_{c}-W_{s}\right)$ and $W_{c}$ is the potential value at the center of the torus. Thus, the enthalpy is analytically known throughout the torus. The values of gas pressure (from the polytropic relation), magnetic pressure $\left(p_{m}=p / \beta\right.$, where $\beta$ is a chosen parameter), magnetic field $\left(B^{2}=24 \pi p_{m}\right)$ and temperature (from the perfect-gas relation) immediately follow. This torus emits thermal synchrotron radiation, following the prescription given in [29]. We note that the term accretion may be misleading given that our model is stationary. However, we consider this torus as a simple model for an instantaneous snapshot of a more realistic time-evolving accretion flow, so we keep referring to it as an accretion torus. The torus model is fully described by the choice of a particular background spacetime plus the choice of a set of seven astrophysical parameters. These are the torus constant angular momentum $\ell$ and inner radius $r_{\text {in }}$ (fixing these two parameters sets the outer radius of the torus), the inclination of the observer $\theta$, the torus central electron number density $n_{c}$ and temperature $T_{c}$, the polytropic exponent $k$ relating pressure and enthalpy, and the gas-to-magnetic pressure ratio $\beta$. Among these astrophysical parameters, only $r_{\text {in }}$ will be varied. The other are fixed to the values given in Table II and were chosen to give reasonable values of fluxes as compared to millimeter observed data of Sgr $\mathrm{A}^{*}$, and to get a rather compact structure (i.e. not a very extended torus but rather a

TABLE II. Torus model astrophysical parameters (the spacetime is not considered here). The value of $r_{\text {in }}$ is slightly varied from spacetime to spacetime to keep the same angular size of the structure, so only an approximate value is given here.

\begin{tabular}{lcc}
\hline \hline Parameter & & Value \\
\hline Inner radius & $r_{\text {in }}$ & $\approx 5.5 M$ \\
Angular momentum & $\ell$ & $3.6 M$ \\
Inclination & $\theta$ & $85^{\circ}$ \\
Central density $\left(\mathrm{cm}^{-3}\right)$ & $n_{c}$ & $6.3 \times 10^{6}$ \\
Central electron temperature $(\mathrm{K})$ & $T_{c}$ & $5.3 \times 10^{10}$ \\
Polytropic exponent & $k$ & $5 / 3$ \\
Gas-to-magnetic pressure ratio & $\beta$ & 10 \\
\hline \hline
\end{tabular}




\section{Kerr BL configuration I}
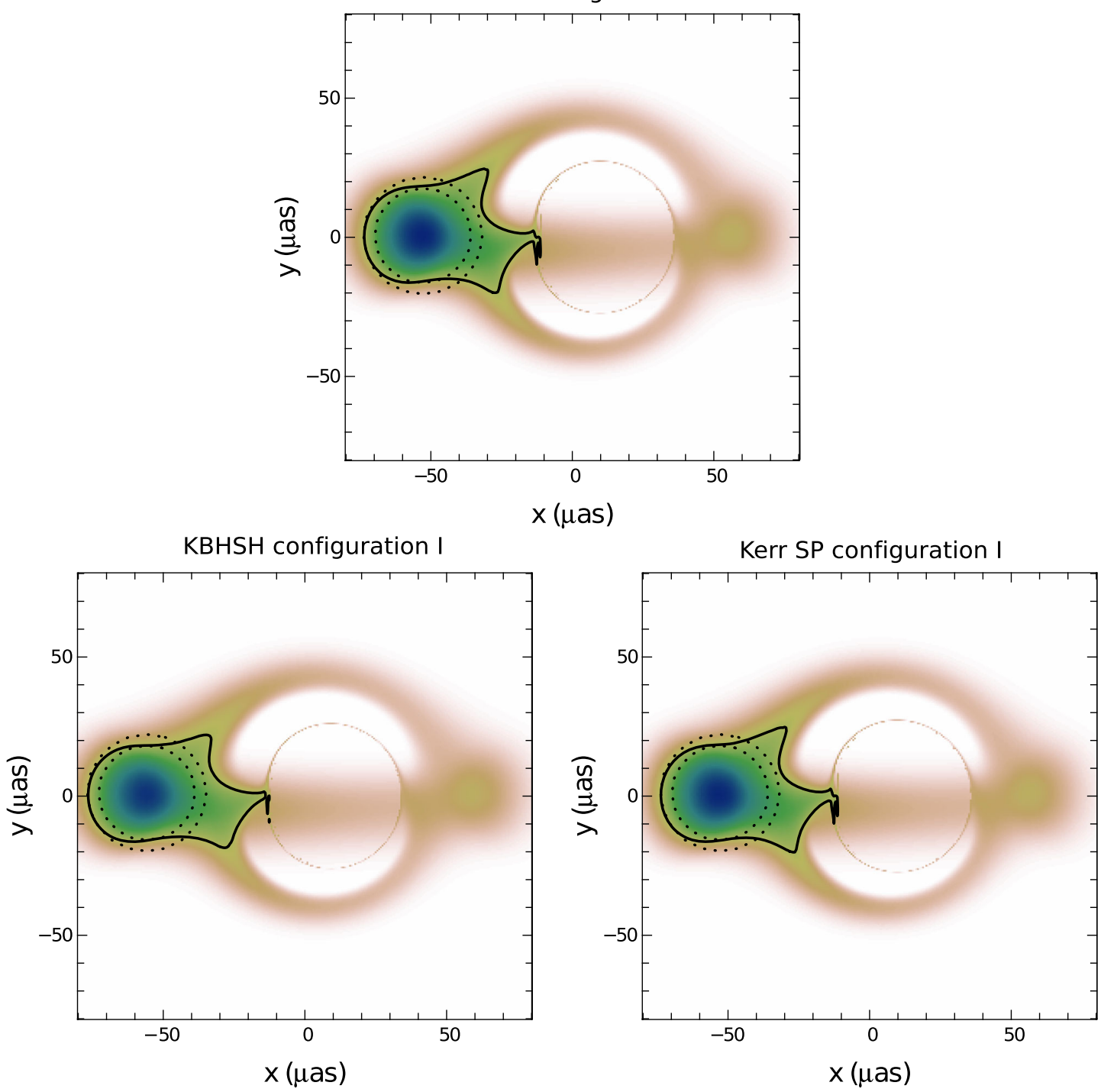

FIG. 5. Configuration I images. Upper panel: Image at $230 \mathrm{GHz}$ of the torus model surrounding the Kerr BH of configuration I, computed with an analytical BL metric. All images in this article are represented in inverse colors: high intensity is in dark blue; low intensity is in yellow/orange. The dotted circles show the $1 \sigma$ upper and lower confidence limits for the intrinsic angular size of the emitting zone [42]. The solid black contour encompasses the region of the accretion flow emitting 50\% of the total flux: it is considered as an order-zero approximation of the size of the emitting region. The image has thus a reasonable angular size provided that the solid contour approximately lies within the dotted circles, which is the case here and in all other figures of the article. Lower left: The corresponding KBHSH setup (same ADM mass and same angular momentum). Lower right: The same image as in the upper panel, but using a numerical Kerr spacetime described with SP coordinates.

structure extending over a small radial distance). For one given spacetime, the inner radius is fixed such that the (SP) radial extent of the structure (outer radius minus inner radius) is close to $20 M$. This ensures to obtain an emitting structure with an angular size satisfying the constraint imposed by the first EHT data [42]. Thus, we choose to vary $r_{\text {in }}$ from spacetime to spacetime in order to maintain an approximately constant angular size of the structure as observed from Earth. Note, however, that the range of variation of $r_{\text {in }}$ is very small and that this quantity always stays close to $r_{\text {in }} \approx 5.5 M$.
The Gyoтo code allows us to ray trace photons from a distant observer and integrate the radiative transfer equation through the optically thin synchrotron-emitting accretion torus, thus producing a map of specific intensity, i.e. an image. We consider an observed frequency of $230 \mathrm{GHz}$ for the ray-traced photons, corresponding to the frequency used for the early EHT science [42]. The aim of this section is to compare each $\mathrm{KBHsSH}$ image to the image of the comparable Kerr configuration, i.e. that having the same ADM mass $M$ and the same total angular momentum $J$ (cf. Sec. II). 
As a first check, we have verified that Kerr BH images computed with SP coordinates were indistinguishable from Kerr images computed with the more standard BoyerLindquist (BL) coordinates. Figure 5 (upper and lowerright panels) shows that this is indeed so: the comparable Kerr image of configuration I computed using the numerical solution in SP coordinates differs by only $\approx 0.7 \%$ with respect to the same image computed using the analytical solution in Boyer-Lindquist coordinates.

The lower-left and upper panels of Fig. 5 show the raytraced images of the KBHSH and comparable Kerr BH of configuration I. The KBHSH and Kerr images are very similar, which is not surprising given that the KBHSH of configuration I was chosen to be very Kerr-like. The flux difference between the KBHSH and Kerr images is $\approx 0.04 \%$, which is vanishingly small as far as spectral observations are concerned. It may seem surprising that this flux ratio is actually smaller than the ratio between the analytical Kerr BL and numerical Kerr SP spacetimes discussed above. This fact is explained by two causes. First, the Kerr BL and Kerr SP spacetimes use different coordinates. This introduces a numerical error that is not present when comparing Kerr SP and KBHSH spacetimes that use the same coordinates. Second, the average specific intensity ratio, when comparing pixel by pixel, is of $0.5 \%$ for the Kerr BL/SP comparison and $4.5 \%$ for the Kerr SP/KBHSH comparison. The flux ratio is different, because most of the flux actually comes from the center of the torus, and in these regions, the numerical error due to the change of coordinates between Kerr BL and SP dominates over the difference between the Kerr SP and KBHSH spacetimes. Arguably the most interesting feature in such strong-field image is the thin ring of illuminated pixels at the center of the image, the photon ring. The KBHSH and Kerr photon rings are very similar in shape, but their sizes differ by $\approx 5 \%$. The recent study of [43] discusses the current measured error on the ratio $M / D$ of the mass of Sgr A* over its distance, and shows that it is of $\approx 6 \%$. Moreover, this study advocates that EHT data could lead to a constraint of the photon ring angular size (the most advanced goal of the EHT) with a precision of $\approx 10 \%$. The size difference of $5 \%$ of the KBHSH and Kerr photon rings that we report here is thus most probably unobservable.

Figure 6 shows the images of the accretion torus surrounding the KBHSH and comparable Kerr solution of configuration II. The flux difference between the KBHSH and Kerr images is $\approx 1.5 \%$, which is still very small with respect to the error bars of spectral observations at $230 \mathrm{GHz}$. The first difference of the KBHSH image with respect to Kerr is the fact that there are two edges in the intensity distribution: there is a brighter ring at the center of the image that looks like a distorted Kerr photon ring (the left part is visibly different from a portion of a circle), and inside this first ring, a second, fainter one. The interpretation of these two features is helped by considering the shadows in Fig. 5 of [26]. These images show clearly that as the spacetime becomes more non-Kerr-like, a region develops around the shadow that is affected by intense and complex lensing effects (this is particularly clear in the third row from the top in Fig. 5 of [26]). In the following we will call this region the hyperlensed region, and its outer boundary the lensing ring (see Fig. 7). The brighter ring in the left panel of our Fig. 6 is this lensing ring, while the fainter ring is the photon ring. We have checked that photons forming the photon ring approach closer to the event horizon (in radial coordinate) than photons forming the lensing ring. This is the expected behavior given that the
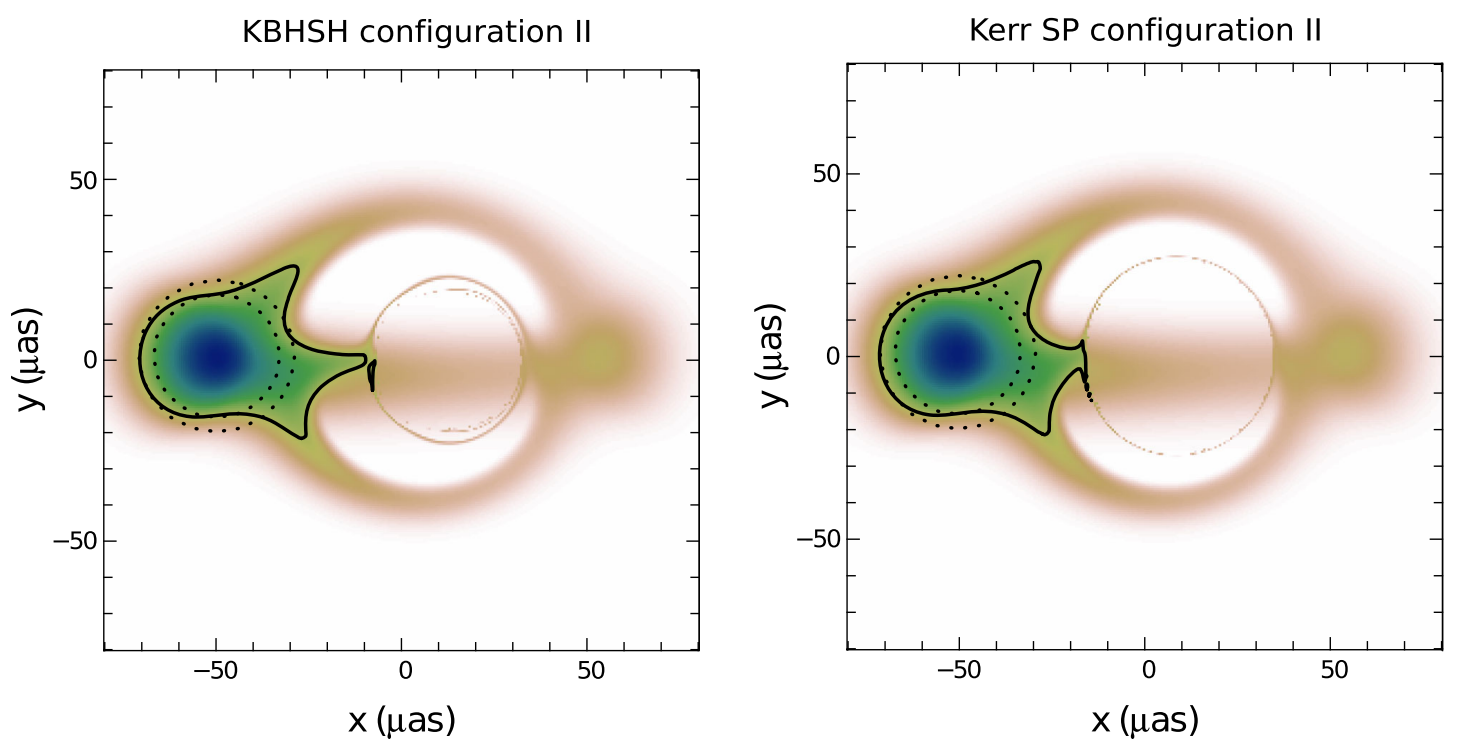

FIG. 6. Configuration II images. Same as in Fig. 5. Left: Image of an accretion torus surrounding the KBHSH of configuration II. Right: Same image for the comparable Kerr case. 
$\mathrm{KBHSH}$ configuration II

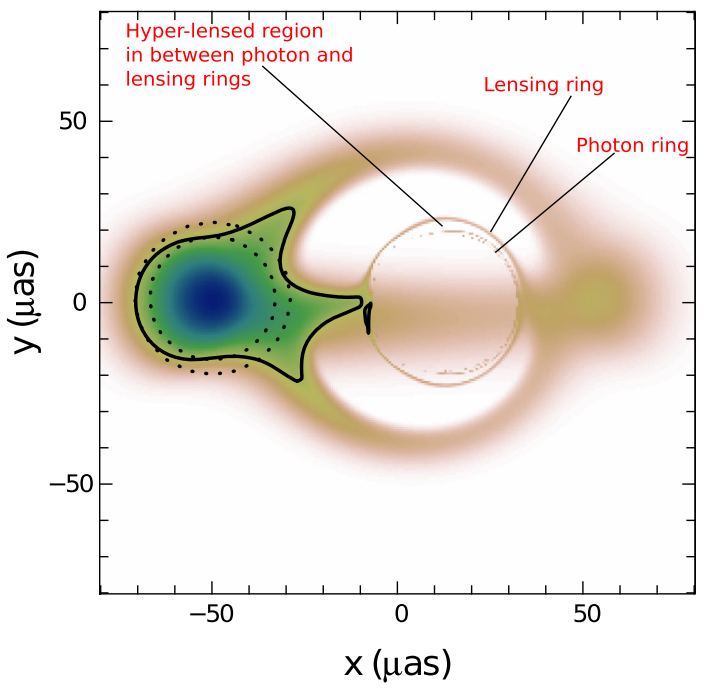

FIG. 7. Illustration of the various interesting regions in the image: The lensing ring is the outermost ring; it is the outer boundary of the hyperlensed region. The left part of the lensing ring is visibly distorted with respect to a Kerr photon ring. The inner ring is the photon ring. It is both the inner boundary of the hyperlensed region and the outer boundary of the shadow.

photon ring is the projection of the innermost photon orbit and marks the innermost limit a photon can visit without falling into the event horizon. It is very probable that EHT would detect only the brighter lensing ring, so we will focus on it in the following. The KBHSH lensing ring is distorted, in the sense that it does not look like the photon ring of any Kerr BH (see [44] for an overview of Kerr photon rings). However, this distortion is extremely tiny and would most

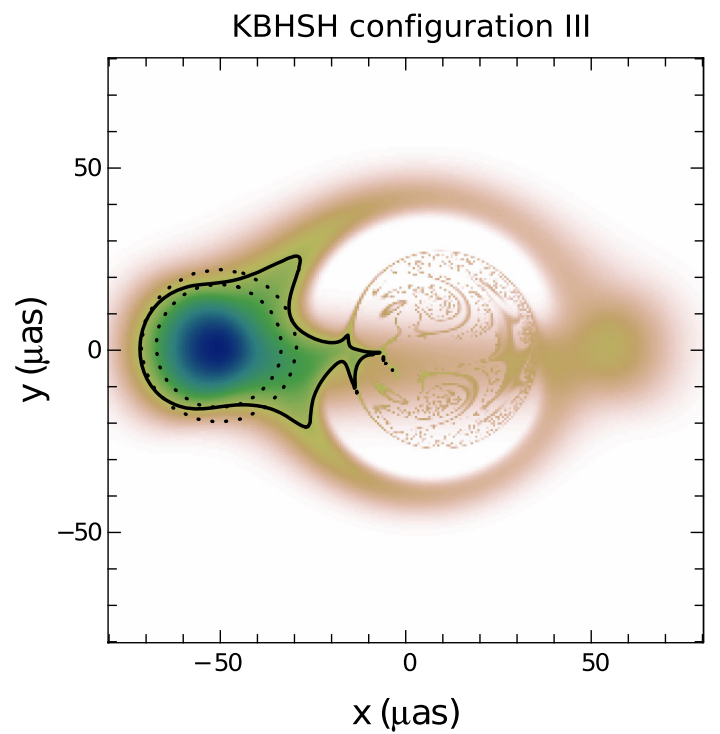

probably be unnoticed by EHT observations. Still, the $\mathrm{KBHSH}$ lensing ring is smaller in angular size, as compared to the Kerr photon ring. The difference in angular size between the KBHSH lensing ring and Kerr photon ring reaches $20 \%$, which is bigger than the error on the ratio $M / D$ as discussed above, and also bigger than the foreseen precision of the EHT data for constraining the photon ring angular size of Sgr A*. Consequently, should the EHT be capable of giving a constraint on the angular size of the photon ring of Sgr $\mathrm{A}^{*}$ to within $\approx 10 \%$ and should this value be too small to be compatible with a Kerr BH of mass $M$ at distance $D$, this would support the existence of an alternative compact object such as a KBHSH at the Galactic center. Note that the comparable Kerr BH of configuration II has a spin of $j_{\mathrm{ADM}}=0.85$. A Kerr BH with spin $j_{\mathrm{ADM}}=$ 0.999 would have a photon ring smaller by approximately $3 \%$ [44], so the difference of $20 \%$ between the Kerr and KBHSH ring angular sizes cannot be accounted for by varying the Kerr $\mathrm{BH}$ spin.

Finally, Fig. 8 shows images of our accretion torus model in the KBHSH and comparable Kerr spacetimes of configuration III. The flux difference between the two images is $\approx 4 \%$, still smaller than the observational error bars. The most striking feature of the left panel in this image is the central "noisy" region, full of radiation. This is the hyperlensed region. Its outer boundary, the lensing ring, nearly coincides with the comparable Kerr photon ring, and is bigger and less distorted than the lensing ring of the KBHSH of configuration II. This is in agreement with the findings of [26]. Why is the hyperlensed region of the configuration III KBHSH "noisy" while the hyperlensed region of its configuration II counterpart is empty of radiation? Because of the very small angular size of the

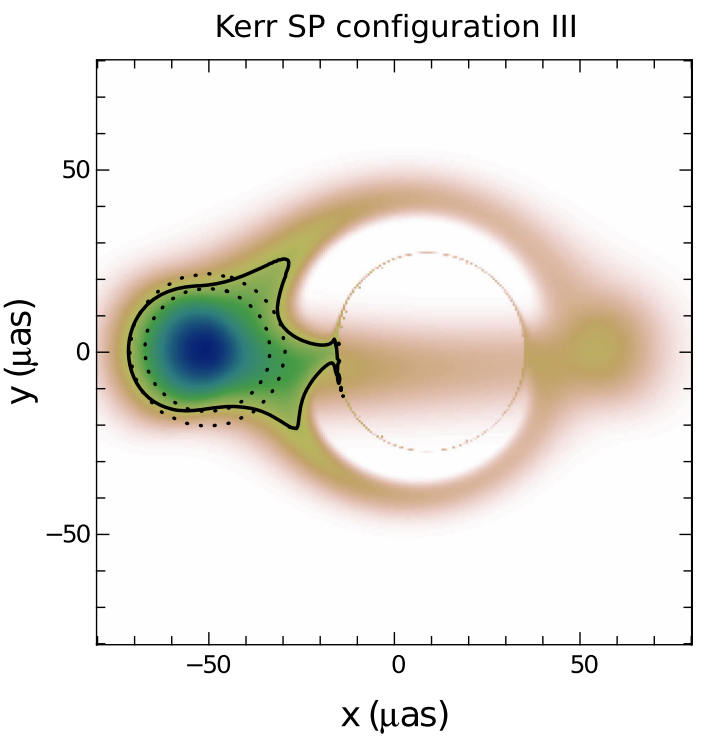

FIG. 8. Configuration III images. Same as in Fig. 5. Left: Image of an accretion torus surrounding the KBHSH of configuration III. Note that the outer boundary of the central "noisy" region is not a photon ring, it is a lensing ring (see text for details). Right: Same image for the comparable Kerr case. The Kerr photon ring is very similar to the KBHSH lensing ring. 
hyperlensed region in configuration II. Photons from inside the hyperlensed region will carry a non-negligible amount of radiation provided they visit the central regions of the accretion torus where most of the radiation is produced. In configuration II, no photon forming the inside of the hyperlensed region visits these central parts of the torus. Only photons forming the lensing ring do so, because the lensing ring corresponds to extremely bent photons and a large region of spacetime (including the inner parts of the torus) is projected to this thin ring of pixels, which is thus bright. On the contrary, many photons from the much bigger hyperlensed region of the KBHSH of configuration III visit the central parts of the torus thus leading to a lot of radiation being located in this region of the image. The $\mathrm{BH}$ shadow is not visible in the left panel of our Fig. 8. It is actually so small in angular size (see Fig. 3) that it is nearly completely erased by the radiation emitted by the part of the torus located in between the $\mathrm{BH}$ and the observer. The photon ring of this spacetime, being the outer boundary of the shadow, is invisible. In this spacetime, the important features, as far as EHT observations are concerned, are the hyperlensed region and lensing ring. The shadow and photon ring are not interesting observationwise.

The hyperlensed region with a large angular scale is a very interesting feature of the KBHSH spacetime of configuration III because it may also lead to observational difference, for different reasons than for the KBHSH of configuration II. The obvious difference between the two panels of Fig. 8 is the fact that there is flux all around the hyperlensed region in the KBHSH spacetime, while the shadow of the Kerr spacetime is free of radiation, except for the radiation emitted in the foreground by the part of the torus in between the $\mathrm{BH}$ and the observer. In the KBHSH spacetime, $\approx 13 \%$ of the total flux is located in the hyperlensed region. In the Kerr spacetime, $\approx 10 \%$ of the total flux is located in the shadow, due to emission in the foreground. Thus, approximately $3 \%$ of the total flux of the KBHSH image of configuration III is a "hairy flux," i.e. due to the hyperlensing effects specific to the KBHSH spacetime. It is probable that an algorithm (see e.g. $[43,45]$ ) trying to detect a Kerr BH shadow on an image similar to the left panel of Fig. 8 would not converge because there is nowhere in the image a photon-ring-like structure: there is no edge (i.e. strong and localized gradient) in the intensity distribution.

\section{FINAL REMARKS}

The perspective of the near-future EHT observations of Sgr A* makes it very timely to study the observable counterparts of compact objects alternative to the Kerr $\mathrm{BH}$. Among the many such objects, $\mathrm{KBHsSH}$ are particularly interesting because (1) they are exact solutions of Einstein field equations, (2) they only necessitate the addition of a scalar field, a rather ubiquitous object in theoretical physics, with the Higgs boson being an example of a fundamental scalar field in nature, and (3) they do not imply adding any astrophysically unclear elements (like the thin shell of gravastars [46]).

This article shows that KBHsSH might be observationally differentiated with respect to Kerr BHs by using EHT observations. A too Kerr-like KBHSH would obviously be impossible to differentiate, as our configuration I illustrates. However, for sufficiently non-Kerr-like KBHSH, we have highlighted two features that may allow making an observational difference. The first such feature illustrated by our configuration II is linked to the angular size of the photon/ lensing rings. The lensing ring is a specific feature of KBHsSH spacetimes defined in Sec. IV, which would observationally be interpreted as a Kerr photon ring. A sufficiently non-Kerr-like KBHSH of the same mass and spin as a Kerr $\mathrm{BH}$ has a lensing ring smaller in angular size than the photon ring of the comparable Kerr image. This size difference reaches $\approx 20 \%$ in our configuration II, which is sufficient to be detectable by EHT data and to be nondegenerate with a Kerr BH. The increasing nonKerrness of a KBHSH spacetime is accompanied by the development of the central hyperlensed region (also defined in Sec. IV), the outer boundary of which is the lensing ring. This hyperlensed region is the second feature that may allow differentiating $\mathrm{KBHsSH}$ from Kerr BHs. Its outer boundary is rather close to the photon ring of a comparable Kerr BH for very non-Kerr-like $\mathrm{KBHsSH}$. As a consequence, the shadow region of a Kerr spacetime, which is characterized by an edge in the intensity distribution, is replaced by a central "noisy" region without an edge. With this edge in intensity distribution being the signal that algorithms investigating such images look for, it is likely that such algorithms would fail finding a shadow region in a KBHSH spacetime such as our configuration III.

Finally, whereas $\mathrm{KBHsSH}$ are certainly an interesting theoretical model for phenomenological deviations from the Kerr paradigm, one may wonder about their realization as astrophysical objects. An obvious necessary condition is the existence of appropriate scalar fields in nature, as discussed in the Introduction, either as fundamental fields, or, eventually, as a coarse graining of more fundamental degrees of freedom. Another central point is the dynamical stability of these configurations. In this respect, we would like to stress that the vacuum Kerr BH (with no scalar field excited) is an unstable solution of the model (1). ${ }^{2}$ Low frequency scalar modes trapped in the vicinity of the $\mathrm{BH}$ trigger a superradiant instability, that grows hair around the BH. Recently, the nonlinear development of this superradiant instability was shown, in toy models where electric charge is taken as a surrogate for rotation, to lead to a hairy

\footnotetext{
${ }^{2}$ The instability of Kerr BHs against low frequency modes of bosonic fields was first discussed by Press and Teukolsky in the setup of a Kerr BH surrounded by a mirror [47]. Subsequently, Damour et al. found that the confining mechanism provided by the mirror is naturally present if the bosonic field is massive [48]. See [49] for an overview of superradiant instabilities.
} 
$\mathrm{BH}[50,51]$. What really happens in the Kerr case is still an open issue, but it seems plausible that some $\mathrm{KBHsSH}$ play a role, either as long-lived transient states, or even as final states in the nonlinear development of this instability. A reasonable expectation is that there are different types of (in)stabilities in the full domain of existence of $\mathrm{KBHsSH}$, with different decay time scales. Efforts to understand these issues in detail are currently under way.

\section{ACKNOWLEDGMENTS}

We would like to thank P. Cunha and H. Rúnarsson for discussions. C. H. and E. R. acknowledge funding from the FCT-IF programme. This work was partially supported by the H2020-MSCA-RISE-2015 Grant No. StronGrHEP690904, by the CIDMA Project No. UID/MAT/04106/ 2013, and by the ANR Grant No. 12-BS01-012-01.
[1] R. Narayan and J.E. McClintock, in General Relativity and Gravitation: A Centennial Perspective, edited by A. Ashtekar, B. K. Berger, J. Isenberg, and M. MacCallum (Cambridge University Press, Cambridge, England, 2015).

[2] B. P. Abbott et al. (Virgo and LIGO Scientific Collaborations), Phys. Rev. Lett. 116, 061102 (2016).

[3] E. Berti et al., Classical Quantum Gravity 32, 243001 (2015).

[4] B. Carter, Phys. Rev. Lett. 26, 331 (1971).

[5] S. W. Hawking, Commun. Math. Phys. 25, 152 (1972).

[6] P. T. Chrusciel, J. L. Costa, and M. Heusler, Living Rev. Relativ. 15, 7 (2012).

[7] R. P. Kerr, Phys. Rev. Lett. 11, 237 (1963).

[8] T. Johannsen, Phys. Rev. D 87, 124017 (2013).

[9] V. Cardoso, P. Pani, and J. Rico, Phys. Rev. D 89, 064007 (2014).

[10] M. Ghasemi-Nodehi and C. Bambi, Eur. Phys. J. C 76, 290 (2016).

[11] L. Rezzolla and A. Zhidenko, Phys. Rev. D 90, 084009 (2014).

[12] R. Konoplya, L. Rezzolla, and A. Zhidenko, Phys. Rev. D 93, 064015 (2016).

[13] C. A. R. Herdeiro and E. Radu, Phys. Rev. Lett. 112, 221101 (2014).

[14] C. A. R. Herdeiro and E. Radu, Int. J. Mod. Phys. D 23, 1442014 (2014).

[15] C. Herdeiro and E. Radu, Classical Quantum Gravity 32, 144001 (2015).

[16] F. Schunck and E. Mielke, Classical Quantum Gravity 20, R301 (2003).

[17] S. L. Liebling and C. Palenzuela, Living Rev. Relativ. 15, 6 (2012).

[18] O. Chodosh and Y. Shlapentokh-Rothman, arXiv: 1510.08025 .

[19] B. Kleihaus, J. Kunz, and S. Yazadjiev, Phys. Lett. B 744, 406 (2015).

[20] C. A. R. Herdeiro, E. Radu, and H. Rúnarsson, Phys. Rev. D 92, 084059 (2015).

[21] C. Herdeiro, E. Radu, and H. Rúnarsson, Classical Quantum Gravity 33, 154001 (2016).

[22] A. Arvanitaki, S. Dimopoulos, S. Dubovsky, N. Kaloper, and J. March-Russell, Phys. Rev. D 81, 123530 (2010).

[23] H. Falcke, F. Melia, and E. Agol, Astrophys. J. 528, L13 (2000).
[24] S. Doeleman et al., arXiv:0906.3899.

[25] A. E. Broderick, T. Johannsen, A. Loeb, and D. Psaltis, Astrophys. J. 784, 7 (2014).

[26] P. V. P. Cunha, C. A. R. Herdeiro, E. Radu, and H. F. Rúnarsson, Phys. Rev. Lett. 115, 211102 (2015).

[27] A. Bohn, W. Throwe, F. Hébert, K. Henriksson, D. Bunandar, M. A. Scheel, and N. W. Taylor, Classical Quantum Gravity 32, 065002 (2015).

[28] F. H. Vincent, T. Paumard, E. Gourgoulhon, and G. Perrin, Classical Quantum Gravity 28, 225011 (2011).

[29] F. H. Vincent, W. Yan, O. Straub, A. A. Zdziarski, and M. A. Abramowicz, Astron. Astrophys. 574, A48 (2015).

[30] F. H. Vincent, Z. Meliani, P. Grandclément, E. Gourgoulhon, and O. Straub, Classical Quantum Gravity 33, 105015 (2016).

[31] P. Grandclément, C. Somé, and E. Gourgoulhon, Phys. Rev. D 90, 024068 (2014)

[32] J. Balakrishna, E. Seidel, and W.-M. Suen, Phys. Rev. D 58, 104004 (1998).

[33] http://gravitation.web.ua.pt/index.php?q=node/416.

[34] C. A. R. Herdeiro and E. Radu, Int. J. Mod. Phys. D 24, 1544022 (2015).

[35] http://gyoto.obspm.fr.

[36] S. Gillessen, F. Eisenhauer, S. Trippe, T. Alexander, R. Genzel, F. Martins, and T. Ott, Astrophys. J. 692, 1075 (2009).

[37] M. Grould, T. Paumard, and G. Perrin, Astron. Astrophys. 591, A116 (2016).

[38] http://www.lorene.obspm.fr.

[39] http://luth.obspm.fr/luthier/grandclement/kadath.html.

[40] W. Schonauer and R. Weiß, J. Comput. Appl. Math. 27, 279 (1989).

[41] S. S. Komissarov, Mon. Not. R. Astron. Soc. 368, 993 (2006).

[42] S. S. Doeleman et al., Nature (London) 455, 78 (2008).

[43] D. Psaltis, F. Özel, C.-K. Chan, and D. P. Marrone, Astrophys. J. 814, 115 (2015).

[44] C.-K. Chan, D. Psaltis, and F. Özel, Astrophys. J. 777, 13 (2013).

[45] T. Johannsen, A. E. Broderick, P. M. Plewa, S. Chatzopoulos, S. S. Doeleman, F. Eisenhauer, V. L. Fish, R. Genzel, O. Gerhard, and M. D. Johnson, Phys. Rev. Lett. 116, 031101 (2016).

[46] P. O. Mazur and E. Mottola, Proc. Natl. Acad. Sci. U.S.A. 101, 9545 (2004). 
[47] W. H. Press and S. A. Teukolsky, Nature (London) 238, 211 (1972).

[48] T. Damour, N. Deruelle, and R. Ruffini, Nuovo Cimento A 15, 257 (1976).

[49] R. Brito, V. Cardoso, and P. Pani, Lect. Notes Phys. 906, 1 (2015).
[50] N. Sanchis-Gual, J. C. Degollado, P. J. Montero, J. A. Font, and C. Herdeiro, Phys. Rev. Lett. 116, 141101 (2016).

[51] P. Bosch, S. R. Green, and L. Lehner, Phys. Rev. Lett. 116, 141102 (2016). 